\title{
Position Estimator Employing Kalman Filter for PM Motors Driven with Binary-type Hall Sensors
}

\author{
Dong-Myung Lee ${ }^{\dagger}$
}

\begin{abstract}
Application of vector control scheme for consumer products is enlarging to improve control performance. For the field-oriented control, accurate position detection is essential and generally requires expensive sensors. On the other hand, cost-reduction is important in home appliances, so that binary-type Hall-effect sensors are commonly used rather than using an expensive sensor such as an encoder. The control performance is directly influenced by the accuracy of the position information, and there exist non-uniformities related to Hall sensors in electrical and mechanical aspects, which result in distorted position information. Therefore, to get high-precision position information from low-resolution Hall sensors, this paper proposes a new position estimator consisting of a Kalman filter and feedforward compensation scheme, which generates a linearly changing position signal. The efficacy of the proposed scheme is verified by simulation and experimental results carried out with a 48-pole permanent magnet motor.
\end{abstract}

Keywords: Kalman filter, Position estimation, Hall-effect sensor, Observer

\section{Introduction}

To improve the performance of home appliances like a washing machine, the vector control technique is widely used. Permanent magnet synchronous motor (PMSM) is commonly used for washing machines due to their excellency in efficiency compared to other type motors. The vector controller must know the rotor position continuously with a fine precision. It is known that the control performance of vector controller highly depends on the accuracy of position information [1]. In a typical variable speed application, a high-precision sensor such as an encoder or resolver is used. However, for household appliances, price down is important. Hence, for reasonable control performance with a cost-effective way, Hall sensors with binary-type, which generate square waveform signals that correspond to $\mathrm{N}$ and $\mathrm{S}$ poles in the rotor, are mainly employed [2]. Various studies to obtain precise position information from the low-resolution square wave signals of Hall sensors have been carried [3-7].

[3-5] proposed a position calculation method based on the zero-order Taylor algorithm, and the configuration is simple and easy to implement. However, as the position value is obtained by integrating the average speed and the calculation of average speed is sensitive to inaccuracies in mechanical installation and non-uniformity in electrical parts, the resultant accuracy is insufficient when there exists an error in the average speed. Moreover, the estimated position values are updated by prefixed values in responding to Hall events, for instance specific value such

\footnotetext{
$\dagger$ Corresponding Author: School of Electronic and Electrical Engineering, Hongik University, Seoul, Korea. (dmlee@hongik.ac.kr) Received: October 8, 2015; Accepted: January 25, 2016
}

as $90^{\circ}$ is assigned to the current position value when the rising edge of one of Hall signals occurs. Thus, uneven time interval between two Hall sensor signals, which causes an error in computing the average speed, generates bumps or discontinuities in the position information where Hall events take place. $[6,7]$ presented a position estimation scheme using an observer. The observer method is able to estimate the rotor position in a wide speed range with the same observer's gain. However, as the full-order observer system is based on the mechanical equation of motion, the accuracy of the estimation is influenced by variation of the mechanical parameter such as inertia $(J)$ and by discrepancy in calculated torque. [8] proposed a position observer utilizing a Kalman filter for speed filtering. In [9], PLL (phase locked loop) has been used for processing of Hall sensor signals. In [10], vector cross-product algorithm was used to improve the calculation performance of the difference between real angle and estimated one. Method of adjusting Hall-sensors offset caused by mechanical misalignment was presented. However, the schemes need the information of torque command and estimated torque value, and basic scheme of [10] is the full-order observer. Hence, same as $[6,7], J$ and $T_{e}$ are involved in estimation of rotor angle and algorithm of compensating misalignment.

The driving condition of the washing machine makes it difficult to apply the observer method because the inertia of a machine varies depending on the amount and distribution status of water and laundry. As noted in [11, 12] the washing machine has a procedure to identify the amount of the laundry by measuring the acceleration and/or deceleration times. It means $J$ is not constant and varies according to the amount of the laundry and water. Hence, an additional work such as online estimation of 
inertia is required to get the position information through the full-order position observer. Therefore to solve these problems, this paper proposes a new position estimation technique, which employs a Kalman filter and feedforward scheme. The performance of the proposed algorithm is verified by simulation studies and experimental results.

\section{Position Signals and Detection by Conventional Methods}

The washing machine of this research is operated by a 48-pole SPM (Surface mounted Permanent Magnet) motor. Two Hall-effect sensors are installed in one electrical revolution span inside the rotor, which results in a $90^{\circ}$ electrical angle resolution. The speed and rotor position by using $90^{\circ}$ resolution Hall sensors can be expressed as (1) and (2), respectively. As expressed in (1), the angular speed is obtained by dividing $90^{\circ}(\pi / 2 \mathrm{rad}$.) to the time interval between Hall sensor events $(\Delta T)$. The position is obtained by the linear extrapolation as (2). Where, $T$ means the sampling time, and $\theta_{e \text { hall }}(k)$ represents the calculated electrical rotor angle at the $k$-th sampling point. It should be mentioned that $\pi / 2$ radians, the numerator of (1), is for an ideal case. In actual case, because of non-uniformity existed in sensors and their circuits, flux of magnets, and mechanical installation, the angle span between Hall sensor events does not form an exact $90^{\circ}$.

$$
\begin{gathered}
\omega_{e_{-} \text {hall }}=\pi / 2 / \Delta T \\
\theta_{e_{-} \text {hall }}(k)=\theta_{e_{-} \text {hall }}(k-1)+\omega_{e_{-} \text {hall }} \cdot T
\end{gathered}
$$

Line (1) in Fig. 1, which has a stair-shape, displays the position updated with the form of $90^{\circ} n, n=0,1,2,3$ in response to the rising and falling edges of the Hall events. Where, the locations marked by dots indicate the exact $90^{\circ} n$ positions. As the point of Hall event differs from $90^{\circ} n$ position with $\alpha, \beta$, etc., $\theta_{e_{-} \text {hall }}$ shown as line (2) has bumps where Hall events occur. This research is applied to a washing machine that has a 48-pole outer rotor. Due to

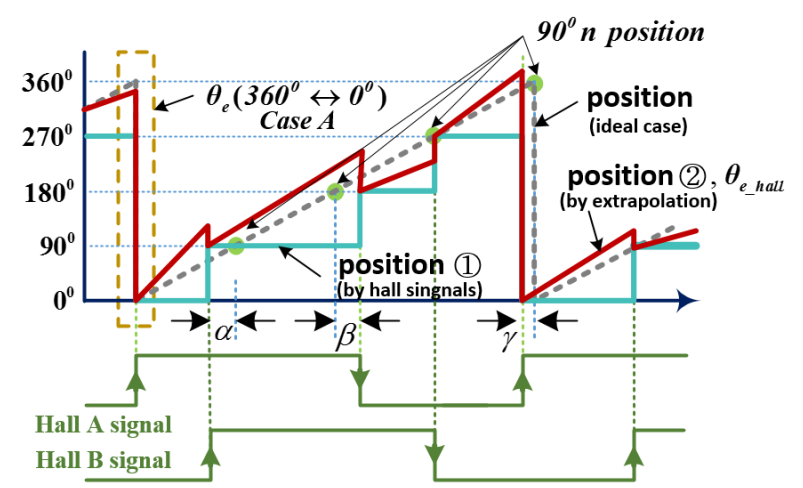

Fig. 1. Position waveforms corresponding to two Hall sensor signals. large numbers of magnets in the rotor and inevitable nonuniformity in the flux of individual magnet, the interval of Hall events is larger or smaller than $90^{\circ}$ randomly. Thus the compensation method adding predetermined offset values for each Hall sensor event can not be applied.

One of the most popular methods of getting linearly changing rotor position is using an observer. The state equation of full-order observer is expressed as (3). ${ }^{\wedge}$ means estimated value. $\omega_{e}, \theta_{e}, T_{L}, T_{e}$, and $P$ represent rotor angular frequency, rotor electrical position, load torque, developed torque, and number of poles, respectively. It is reported that the transfer function of an observer has the form of a low-pass filter about position [10], hence it is used to filter out bumps. However, inaccurately calculated $J$ and $T_{e}$ may degrade the ability of position estimation, and the simulation results of its effects will be shown in Section IV.

$$
\frac{d}{d t}\left[\begin{array}{c}
\hat{\theta}_{e} \\
\hat{\omega}_{e} \\
\hat{T}_{L}
\end{array}\right]=\left[\begin{array}{ccc}
0 & 1 & 0 \\
0 & 0 & -\frac{1}{J} \frac{P}{2} \\
0 & 0 & 0
\end{array}\right]\left[\begin{array}{c}
\hat{\theta}_{e} \\
\hat{\omega}_{e} \\
\hat{T}_{L}
\end{array}\right]+\left[\begin{array}{c}
0 \\
\frac{1}{J} \frac{P}{2} \\
0
\end{array}\right] T_{e}+\left[\begin{array}{c}
l_{1} \\
l_{2} \\
l_{3}
\end{array}\right]\left(\theta_{e_{-} \text {hall }}-\hat{\theta}_{e}\right)
$$

\section{Proposed Method for Position Estimation}

A Kalman filter is a stochastic and optimal recursive algorithm and application of the Kalman filter for estimating position and/or speed has been proposed in many studies especially for sensorless control [13, 14]. However, a research for obtaining linearly changing rotor position signal from binary-type Hall sensors by adopting only a Kalman filter has not been presented as far as the author knows. To prevent incorrect position estimation due to inaccurate system parameters or miscalculation, the proposed scheme employs the Kalman filter algorithm. In addition, a feedforward scheme is devised to cope with large position transition such as from $2 \pi$ to 0 radians or vice versa, denoted as case A in Fig. 1. Moreover, it is reported that similar cases of this research exist in various applications. [15] said that the position signal of a magnetic levitation train without using a linear scaler resembles the line (2), and development of an algorithm to get linearly changing position signal is required. Thus, it is obvious that the proposed scheme can be applied to not only washing machine drives but also many other applications requiring high-precision position signal from low-resolution sensors. The equations of the Kalman filter are expressed as (4) (7). $K$ stands for Kalman filter gain matrix, and $P$ is state covariance matrix. - represents predicted estimate, $k$ and $k$ - 1 denote present value and one step previous value, separately $[16,17]$.

$$
\hat{x}_{k}^{-}=A \hat{x}_{k-1}, P_{k}^{-}=A P_{k-1} A^{T}+Q
$$




$$
\begin{gathered}
K_{k}=P_{k}^{-} H^{T}\left(H P_{k}^{-} H^{T}+R\right)^{-1} \\
\hat{x}_{k}=\hat{x}_{k}^{-}+K_{k}\left(z_{k}-H \hat{x}_{k}^{-}\right) \\
P_{k}=P_{k}^{-}-K_{k} H P_{k}^{-}
\end{gathered}
$$

With the assumption that there is no rotor $\operatorname{speed}\left(\omega_{e}\right)$ variation during the sampling interval $T$ and with the fact that $\omega_{e}=d \theta_{e} / d t$, the equations in the discrete time domain can be given as (8) (11). Where, state variable $x$ consists of $\theta_{e}$ and $\omega_{e}$. Measurement $(z)$ is expressed as (10).

$$
\begin{gathered}
\hat{x}(k)=A \hat{x}(k-1), z(k)=H x(k) \\
\hat{x}(k)=\left[\begin{array}{l}
\hat{\theta}_{e}(k) \\
\hat{\omega}_{e}(k)
\end{array}\right]=\left[\begin{array}{ll}
1 & T \\
0 & 1
\end{array}\right]\left[\begin{array}{l}
\hat{\theta}_{e}(k-1) \\
\hat{\omega}_{e}(k-1)
\end{array}\right] \\
z(k)=\left[\begin{array}{l}
\theta_{e_{-} \text {hall }}(k) \\
\omega_{e_{-} \text {hall }}(\mathrm{k})
\end{array}\right]=\left[\begin{array}{ll}
1 & 0 \\
0 & 1
\end{array}\right]\left[\begin{array}{l}
\theta_{e_{-} \text {hall }}(k) \\
\omega_{e_{-} \text {hall }}(\mathrm{k})
\end{array}\right] \\
A=\left[\begin{array}{ll}
1 & T \\
0 & 1
\end{array}\right], H=\left[\begin{array}{ll}
1 & 0 \\
0 & 1
\end{array}\right] \\
Q=\left[\begin{array}{cc}
\delta_{1}^{2} & 0 \\
0 & \delta_{2}^{2}
\end{array}\right], R=\left[\begin{array}{cc}
\delta_{3}^{2} & 0 \\
0 & \delta_{4}^{2}
\end{array}\right]
\end{gathered}
$$

where, $Q$ and $R$ in (4), (5), and (12) are the covariance matrix of process noise and measurement noise, respectively.

Larger $R$ results in smaller $K_{k}$, which means $\hat{x}_{k}$ has greater weight on $\hat{x}_{k}^{-}$than on $z_{k}$. As a result, the estimate changes smoothly, i.e. high filtering results. On the other hand, higher $Q$ generates larger $P_{k}^{-}$, which results in less filtering effect because $\hat{x}_{k}$ receives more impact from $z_{k}$ than from $\hat{x}_{k}^{-}$. As $Q_{l l}$, and $R_{l l}$ are involved in filtering position, larger $R_{l l}$ and smaller $Q_{11}$ are required to increase the filtering effect. In order to get rid of bumps in the estimated position, high filtering effect is required. However, a position estimator with $R$ and $Q$ matrices corresponding to high filtering effect can not track actual one well especially for the case A having a large angle change. In contrast, an estimator with $R_{l l}$ and $Q_{1 l}$ for small filtering performance can follow the actual position well including case A condition. However, there arises the impossibility of removing bumps due to less filtering result. On the other hand, in the case of the observer, by modifying $\left(\theta_{e \text { hall }}-\hat{\theta}_{e}\right)$ of (3) to $\sin \left(\theta_{e \text { hall }}-\hat{\theta}_{e}\right)$, case $\mathrm{A}$ can be dealt with because $\sin (2 \pi+\alpha)=\sin (\alpha) \approx \alpha$ for small angle $\alpha$. Whereas, that modification worked in the observer scheme can not be applied to the Kalman filter because $\sin \left(\theta_{e_{-} \text {hall }}-\hat{\theta}_{e}\right)$ can not be chosen as the state variable. Hence, to cope with case A, a feedforward scheme is devised in this paper. Fig. 2 shows the overall block diagram of driving system of PM motors with the proposed scheme. As shown in Fig. 2, $\omega_{e \text { hall }}$ and $\theta_{e \text { hall }}$ are obtained by (1) and (2) from incoming Hall events. Through the proposed scheme expressed as (4) (7), filtered $\omega_{e}$ and linearly changing position signal are generated. The

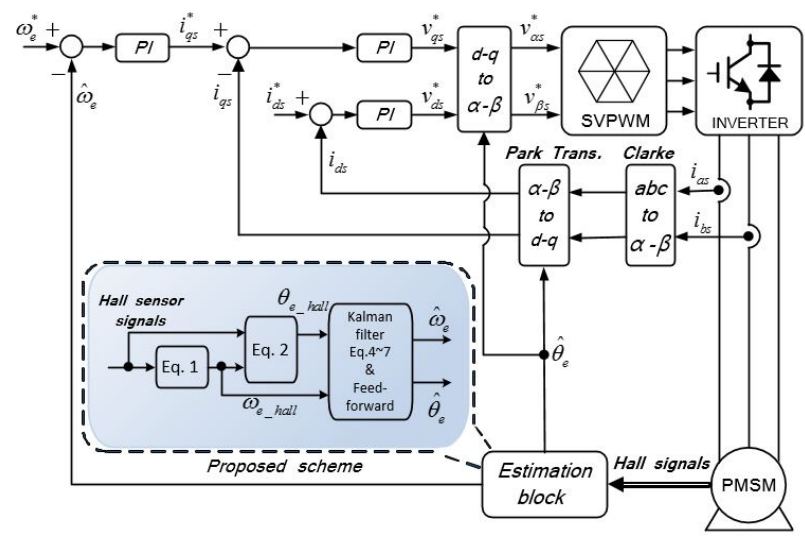

Fig. 2. Block diagram of PM drive with the proposed scheme.

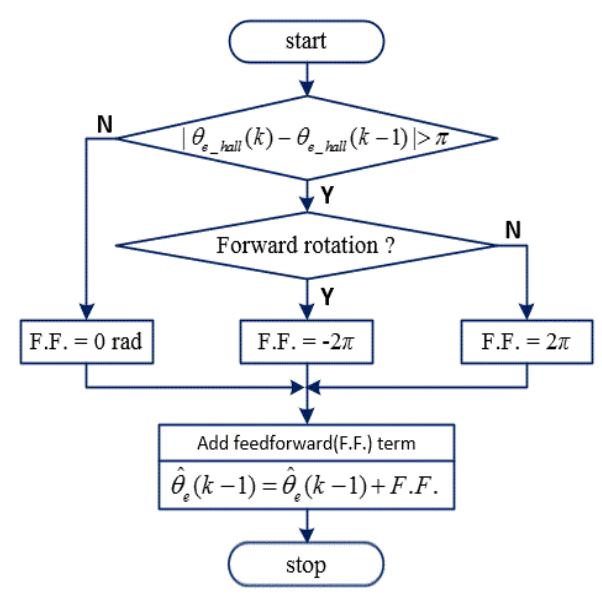

Fig. 3. Flowchart for calculating the feedforward term.

resultant bumpless $\theta_{e}$ is used as the rotor flux angle for $d-q$ transformation. Fig. 3 illustrates the flowchart that determines feedforward (F.F.) value. As shown in Fig. 3, whether case A occurs or not is determined by checking the difference between $\theta_{e_{\_} \text {hall }}(k)$ and $\theta_{e_{\_} \text {hall }}(k-1)$. F.F. value is assigned for $0 \rightarrow 2 \pi$ or $2 \pi \rightarrow 0$ transition conditions otherwise zero. The F.F. value added to is $-2 \pi$ for the forward rotation and $+2 \pi$ for the reverse spin. The F.F. scheme seems so simple but without it the proposed method can not filter out distortions in the position signal.

\section{Verifications}

\subsection{Simulation results of position estimation scheme using full-order observer}

In Section IV, simulation results of conventional method and proposed scheme are shown. Fig. 4 displays the block diagram of the full-order observer scheme expressed in (3). The block of vector cross algorithm done in [7] is not illustrated in Fig. 4, but in the actual implementation taking sine of $\left(\theta_{e_{-} \text {hall }}-\hat{\theta}_{e}\right)$ has been done after the summing 


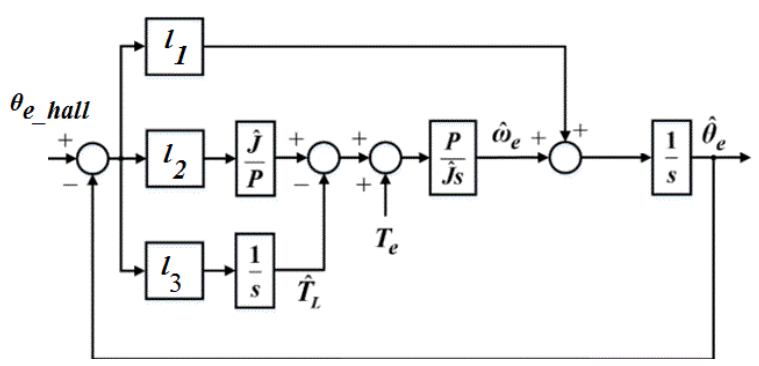

Fig. 4. One of conventional methods that employs the full-order observer.

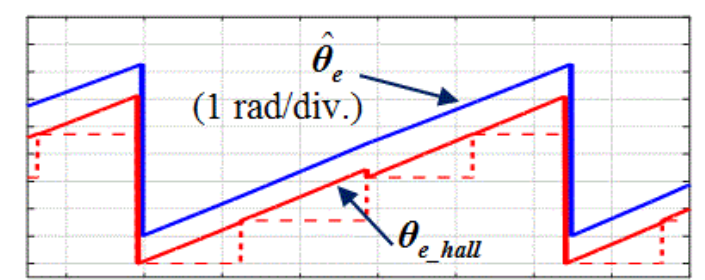

(a)

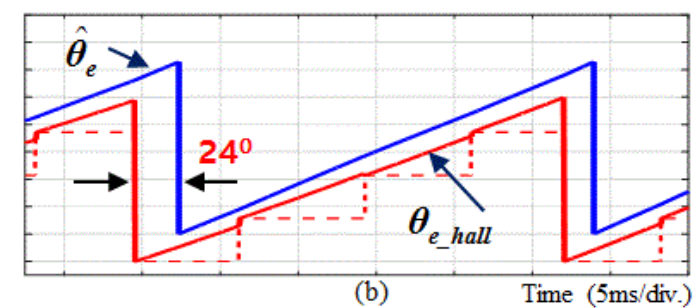

Fig. 5. Simulation results: position signals obtained by fullorder observer with (a) exact system parameters and (b) with $80 \% \mathrm{~J}$ and $85 \% T_{e}$ with respect to actual ones.

junction of $\left(\theta_{e \text { hall }}-\hat{\theta}_{e}\right)$ to cope with the case A shown in Fig. 1.

In Fig. $4, J$ and $T_{e}$ are estimated values, and $T_{e}$ value is obtained by the below equation.

$$
T_{e}=\frac{3}{2} \frac{P}{2} \lambda_{m} i_{q s}
$$

where $P$ denotes number of poles, and $\lambda_{m}$ represents fluxlinkage by permanent magnet. Ferrite-based permanent magnets are known to have a $20 \%$ flux reduction per $100^{\circ} \mathrm{C}$ increase, hence to get accurate $T_{e}$ value online estimation of $\lambda_{m}$ is required.

Fig. 5 shows simulation waveforms of full-order observer with (a) exactly estimated system parameters and with (b) errors in $J$ and $T_{e}$ estimation, respectively. From top to bottom, estimated theta, $\theta_{e \text { hall }}$, and $\theta_{e}$ formed as $90^{\circ} n$ (dotted line) are shown. The washing machine rotates under constant speed and torque condition. Fig. 5(b) illustrates the simulation results carried out with $20 \%$-less $J$ value and $15 \%$-less $T_{e}$ than actual ones. It shows that the observer scheme, which has errors in $J$ and $T_{e}$ computation, results in $24^{0}$ angle difference between actual and
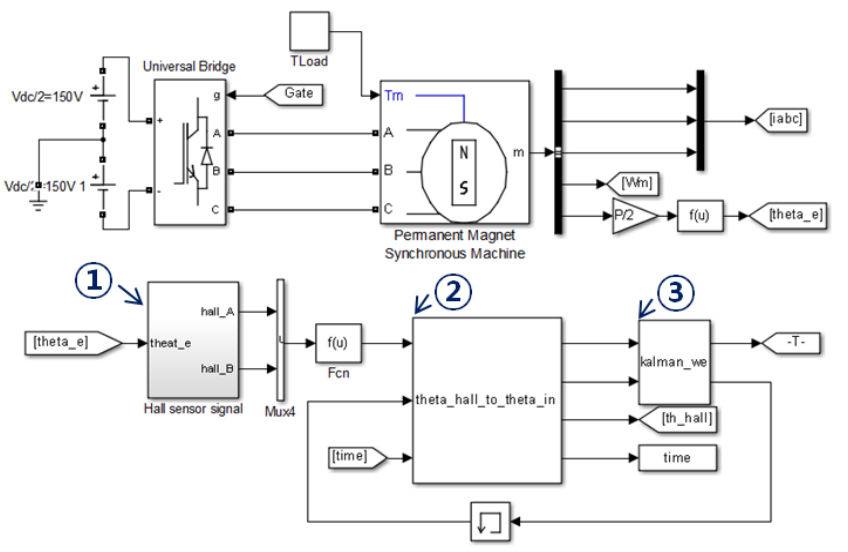

Fig. 6. Simulation block diagram of the proposed scheme.

estimated values, which clearly shows the big angle difference between $\theta_{e}$ hall and estimated $\theta_{e}$. Even in the case of Fig. 5(a) simulated with correct system parameters and exactly calculated $T_{e}$, there was about $3^{0}$ angle discrepancy.

\subsection{Simulation results related to the proposed scheme}

The simulation model of the proposed scheme, which is made by Matlab/Simulink is shown in Fig. 6. To avoid errors in system modeling, devices provided in sympowersystems library of Simulink are actively used such that the SPM motor and the inverter existed in library are used. The simulation study of the full-order order observer has also been conducted using Simulink. In Fig. 6, blocks of controllers for field-oriented control are not displayed due to limited space and less relevant to the proposed scheme.

Block (1) shown in Fig. 6 generates Hall signals A and $\mathrm{B}$, which simulate actual situation such that the high state of each signal is not $180^{\circ}$, by using the rotor position from the motor model. In Block (2), $\theta_{e}$ hall is obtained based on (2), and the process of assigning the feedforward term is conducted.

Whereas as shown in the figure, for obtaining $\theta_{e_{-} \text {hall }}$ instead of $\omega_{e}$ hall, $\hat{\omega}_{e}$ from Block (3) is used. In Block (3), the proposed scheme based on Kalman filter is implemented by s-function of Matlab, and outputs of Block (3) are estimated theta and rotor speed. Specification of the motor used for this simulation studies is summarized in Table 1.

Various simulation results related to the proposed scheme are displayed in Fig. 7. Fig. 7(a) (c) illustrate simulation results obtained without imposing the feedforward scheme. The estimated positions are displayed 1 radian above $\theta_{e}$ hall to discriminate these two waveforms. Fig. 7(a) shows simulation waveforms conducted with $R_{11}$ and $Q_{11}$ having less filtering effect. As shown in Fig. 7(a), the estimated signal goes along with $\theta_{e}$ hall well in all $2 \pi$ range including the case of rapid angle change, but the bumps are not removed at all due to the less filtering performance. In the case of Fig. 7(b), the $R_{11}$ and $Q_{11}$ were 


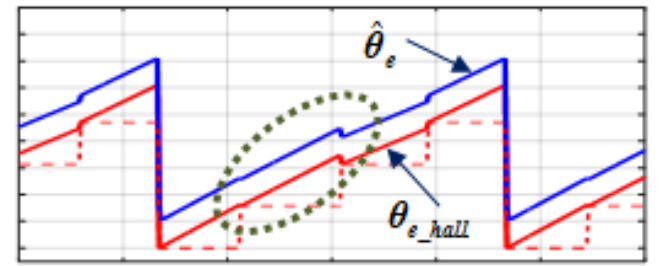

(a)

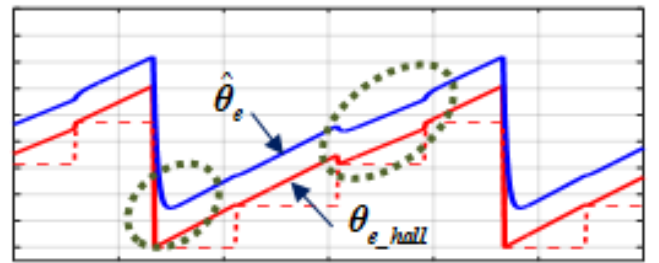

(b)

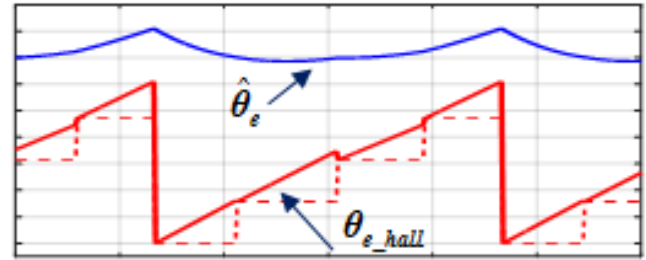

(c)

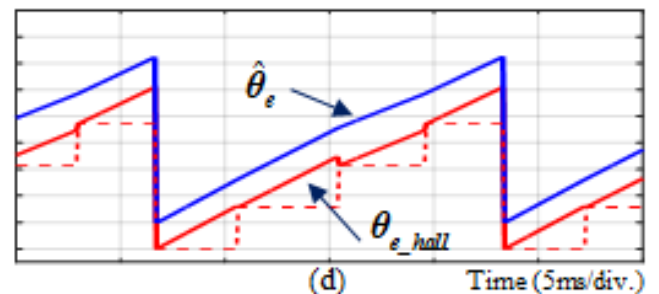

(d)

Fig. 7. Simulation waveforms: (a) with the covariance values for less filtering effect without F.F; (b) with the covariance values for moderate filtering effect without F.F; (c) with the covariance values for high filtering effect without F.F; (d) by the proposed scheme with the same covariance values of Fig. 7 (c) but with feedforward (F.F.) scheme.

chosen to get more filtering effect than those of Fig. 7(a).

As shown inside the circle, these $R$ and $Q$ matrices can not generate the estimated position that keeps up with $\theta_{e}$ hall varying from $2 \pi$ to 0 . Furthermore, filtering result is not good enough to eliminate the discontinuities in the estimated position. The covariance matrices of Fig. 7(c) and $7(d)$ are the same each other, the only difference is whether the feedforward(F.F.) scheme is employed or not. Fig. 7(c) illustrates that the algorithm without F.F. term fails to estimate the position. On the other hand, the proposed scheme having F.F. term with the same $R$ and $Q$ matrices of Fig. 7(c) removes the bumps and generates a linearly changing rotor position in all $2 \pi$ range as displayed in Fig. 7(d). Fig. 8 illustrates simulation results (a) without and (b) with the proposed scheme. From the top to the bottom of the figure, Hall $A \& B$ signals, $\theta_{e}$ signals, and phase current are illustrated. As shown in Fig 8(a) about without the proposed scheme, there exist discontinuities in

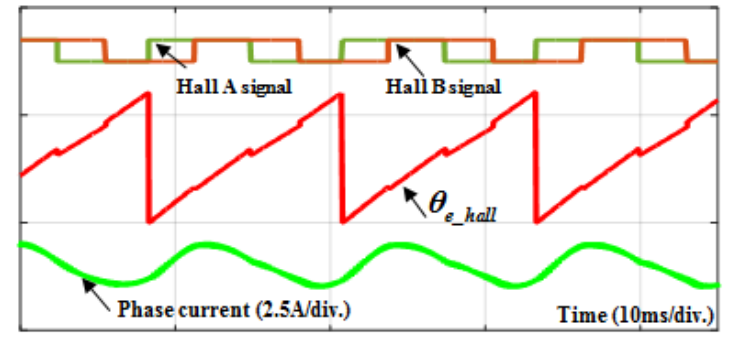

(a)

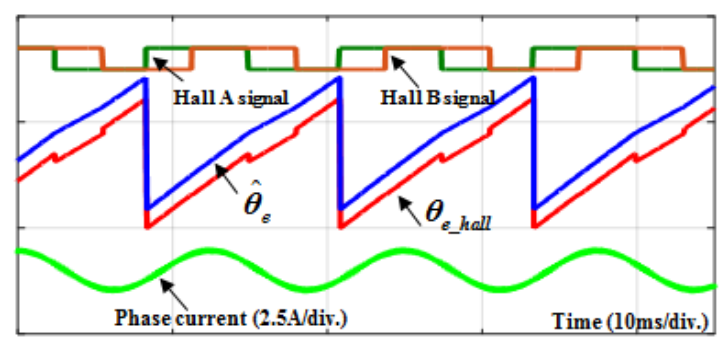

(b)

Fig. 8. Simulation waveforms: (a) with and (b) without the proposed scheme.

rotor position signal and the phase current has distortion a result of bumps in the position signal. In contrast, the theta in Fig. 8(b) obtained by the proposed scheme has no bumps and the resultant phase current is sinusoidal. Hence it shows the performance and efficacy of the proposed scheme. The $\theta_{\text {e_hall }}$ waveform in Fig. $8(\mathrm{~b})$ seems to have smaller distortion than that in Fig. 8(a). The reason is that $\theta_{e_{\text {h hall }}}$ shown in Fig. 8(a) is obtained by (2), but that in Fig. $8(\bar{b})$ is gotten as (14) by using the estimated speed from the Kalman filter. It should be noted again that the simulation conditions of with and without are identical each other, i.e. the position errors in Hall signals are the same each other.

$$
\theta_{e_{-} \text {hall }}(k)=\theta_{e_{-} \text {hall }}(k-1)+\hat{\omega}_{e}(k-1) \cdot T
$$

where, $\hat{\omega}_{e}(k-1)$ represents the estimated rotor speed at the $k$-1 sampling point.

\subsection{Experimental results}

Experiments have been carried out with a 48-pole SPM motors to verify the performance of the proposed scheme. Fig. 9 illustrates an overall experimental setup. The IGBTs in the voltage source inverter supplied by $300 \mathrm{~V}$ dc-link were switched on and off at a frequency of $10 \mathrm{kHz}$ to control the PM motor.

The proposed position estimation scheme has been implemented by TI's TMS320F28335 DSP controller. Two binary Hall sensor signals were fed to the interrupt ports of the DSP chip via an interface circuit and converted to the linearly varying position information by the proposed scheme. 


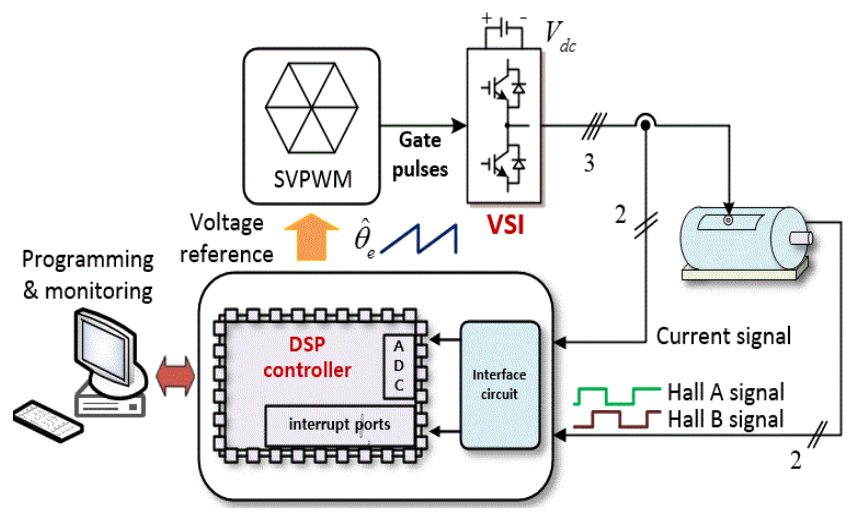

Fig. 9. Experimental setup.

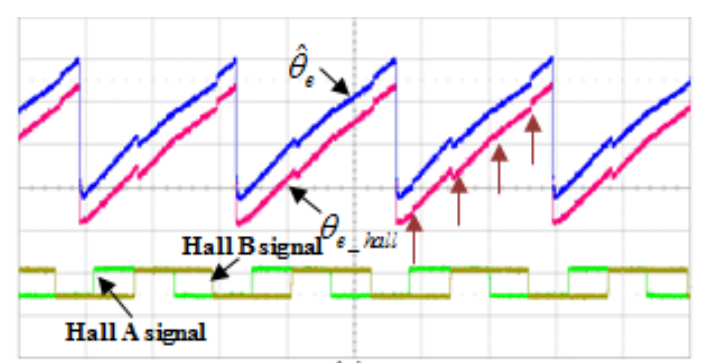

(a)

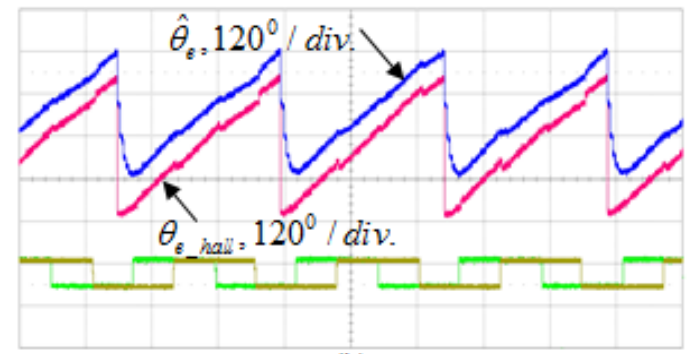

(b)

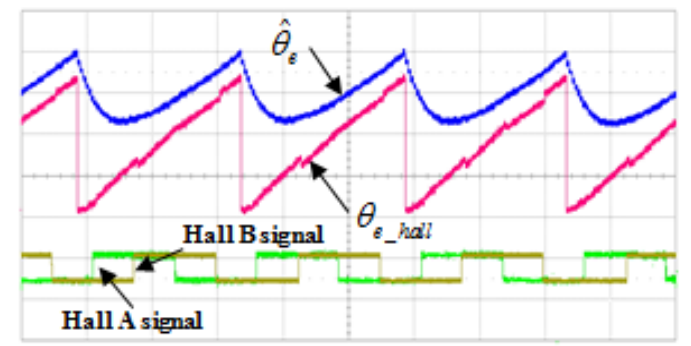

(c)

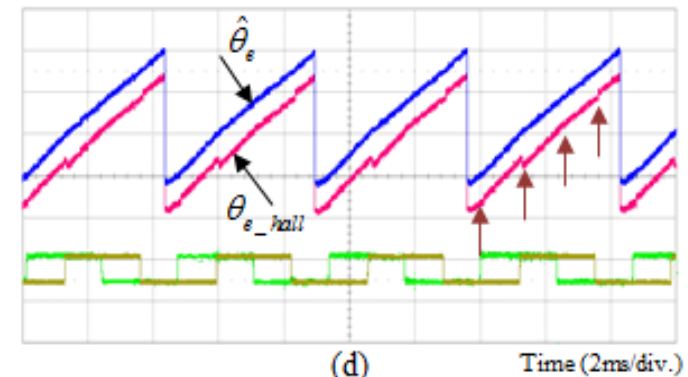

(d)

Fig. 10. Experimental results conducted with the same simulation conditions of (a) Fig. 7a, (b) Fig. 7b, (c) Fig. 7c, and (d) Fig. 7d (the proposed scheme).

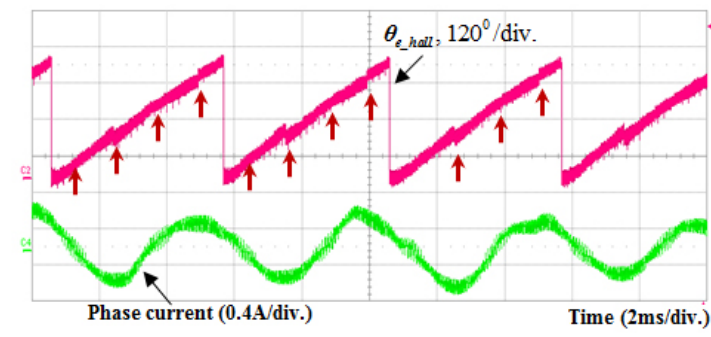

(a)

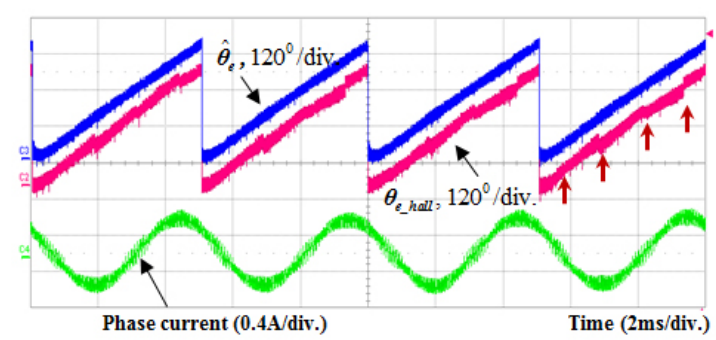

(b)

Fig. 11. Experimental results: position and phase current waveforms when the machine operates at $500 \mathrm{rpm}$ (a) without and (b) with the proposed method.

The $d-q$ voltage references have been converted to gate pulses by using the space vector PWM with the conversion of voltage references in the rotating fame to those in the stationary frame by using the estimated position obtained through the proposed scheme. Fig. 10 shows experimental results carried out with the same simulation conditions of Fig. 7 in which Fig. 10(a) (d) correspond to Fig. 7(a) (d). In Fig. 10, Hall sensor signals, $\theta_{e}$ hall by (2), and the estimated position are illustrated. As appreciated in those figures, $\theta_{e \text { hall }}$ has bumps approximately four times in one electrical cycle. In the real implementation, the first rising edge of Hall A signal occurs not $0^{0}$ but $30^{\circ}$ so that the positions where bumps occur are different from those in the simulation waveforms. It can be seen that experimental and simulation results well agree each other. For instance, Fig. 10(a) showing experimental waveforms and Fig. 7(a) illustrating simulation waveforms under the same condition are almost identical each other, similarly Fig. 10(b) with respect to Fig. 7(b). Fig 10(c) shows that due to the covariance matrices having the high filtering effect the position estimation does not work the same as Fig. 7(c). In contrast, Fig. 10(d), in which $R$ and $Q$ values are identical to those of Fig. 10(c), shows linearly changing position information obtained from the binary-type Hall sensors by employing a Kalman filter with the feedforward scheme. Therefore, it verifies the efficacy of the proposed scheme. Furthermore, experiments to indicate the performance improvement by displaying the phase current waveform have been conducted.

Fig. 11(a) and 11(b) show experimental waveforms without and with the proposed scheme, respectively. Fig 11(a) reveals 
that there are deformations in the position information, and the phase current has distortion as well. Fig. 11(b) clearly displays the elimination of bumps by the proposed scheme and the resultant sinusoidal phase current waveform.

\section{Conclusion}

This paper proposed a new position estimation scheme for PMSM driving system in which the rotor position was detected by low-resolution Hall-effect sensors. The proposed scheme employed the Kalman filter in order to remove the necessity of system parameters and errors caused by inaccurately computed system variables. In addition, the feedforward scheme was devised to have high filtering performance in the entire angle range. Simulation results of proposed scheme as well as conventional method have been illustrated. Validity and effectiveness of the proposed algorithm were verified by simulation and experimental investigations.

\section{Acknowledgement}

This work was supported by the Korea Institute of Energy Technology Evaluation and Planning(KETEP) granted financial resource from the Ministry of Trade, Industry \& Energy, Republic of Korea (No. 20152010103540)

\section{References}

[1] S. Y. Kim, C. C. Choi, K. J. Lee, and W. T. Lee, “An improved rotor position estimation with vector tracking observer in PMSM drives with low resolution Halleffect sensors," IEEE Trans. Ind. Electron., vol. 58, no. 9, pp. 4078-4086, 2011.

[2] J. D. R. Dominguez, A. Navarrete, M. A. Meza, A. G. Loukianov, and J. Cañedo, "Digital sliding-mode sensorless control for surface-mounted PMSM," IEEE Trans. Ind. Informat., vol. 10, no. 1, pp. 137-151, Feb. 2014.

[3] J. Fang, X. Zhou, and G. Liu, "Instantaneous torque control of small inductance brushless DC motor," IEEE Trans. Power Elect., vol. 27, no. 12, pp. 49524964, Dec. 2012.

[4] Z. Feng and P. P. Acarnley, "Extrapolation technique for improving the effective resolution of position encoders in permanent-magnet motor drives," IEEE Trans. Mechatron., vol. 13, no. 4, pp. 410-415, Aug. 2008.

[5] P. B. Beccue, S. D. Pekarek, B. J. Deken, and A. C. Koenig, "Compensation for asymmetries and misalignment in a Hall-effect position observer used in PMSM torque-ripple control,” IEEE Trans. Ind. Appl., vol. 43, no. 2, pp. 560-570, Mar./Apr. 2007.

[6] M. C. Harke, G. D. Donato, F. G. Capponi, T. R. A. Tesch, and R. D. Lorenz, "Disturbance torque and motion state estimation with low-resolution position interfaces using heterodyning observers," IEEE Trans. Indus. Appli., vol. 44, no. 1, pp. 124-134, Jan/Feb. 2008.

[7] A. Yoo, S. K. Sul, D. C. Lee, and C.S. Jun, "Novel speed and rotor position estimation strategy using a dual observer for low-resolution position sensors," IEEE Trans. Power Electron., vol. 24, no. 12, pp. 2897-2906, Dec. 2009.

[8] D. M. Lee, D. C. Lim, and H. J. Ahn, "Position linearisation scheme for permanent magnet synchronous motor drive of washing machine using lowresolution hall sensors," IET Elect. Letters, vol. 51, no. 22, pp. 1765-1767, Oct. 2015.

[9] F. G. Capponi, G. Donato, L. Ferraro, O. Honorati, M. C. Harke, and R. D. Lorenz, "AC brushless drive with low-resolution Hall-effect sensors for surfacemounted PM machines," IEEE Trans. Ind. Appl., vol. 42, no. 2, pp. 526-535, Mar./Apr. 2006.

[10] Z. M. Dalala, Y. H. Cho, and J. H. Lai, "Enhanced vector tracking observer for rotor position estimation for PMSM drives with low resolution Hall-effect position sensors," IEEE Inter. Electric Machines \& Drives Conf., pp. 484-491, 2013.

[11] T. Payne, S. Rice, D. Able, and D. Dickerson, "Electronic washer control including automatic load size determination, fabric blend determination and adjustable washer means," US Patent 5161393A, 1992.

[12] F. Altinier, T. Girotto, and M. Giovagnoni, "Method for estimating the moment of inertia of the rotating unit of a washing machine, and washing machine implementing said method," US Patent 8336378, 2012.

[13] Z. Aydogmus and O. Aydogmus, "A comparison of artificial neural network and extended Kalman filter based sensorless speed estimation," Measurement Elsevier, vol. 63, pp. 152-158, March 2015.

[14] N. K. Quang, N. T. Hieu, and Q. P. Ha, "FPGA-based sensorless PMSM speed control using reduced-order extended Kalman filters," IEEE Trans. Indus. Elect., vol. 61, no. 12, pp. 6574-6582, Dec. 2014.

[15] Y. J. Han, C.Y. Lee, and Y. Sun, "A study on the sensor applications for position detection and guideway monitoring in high speed Maglev," Modern Mech. Engineering, vol. 4, no. 4, pp.165-174, 2014.

[16] Z. Chen, Y. Fu, and C. C. Mi, "State of charge estimation of lithium-ion batteries in electric drive vehicles using extended Kalman filtering," IEEE Trans. Vehicular Technology, vol. 62, no. 3, pp.10201030, March 2013.

[17] H. W. Sim, J. S. Lee, and K. B. Lee, "On-line parameter estimation of interior permanent magnet synchronous motor using an extended Kalman filter," Journal of Electr. Eng. Tech., vol. 9, no. 2, pp. 600608, March. 2014. 


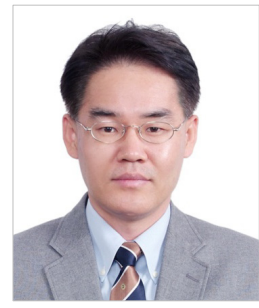

Dong-Myung Lee received his B.S. and M.S. in Electrical Engineering from Hanyang University, Seoul, Korea, in 1994 and 1996, respectively, and his Ph.D. in Electrical and Computer Engineering from the Georgia Institute of Technology, Atlanta, Georgia, USA, in 2004. From 1996 to 2000, he worked for LG Electronics Inc., Seoul, Korea. From 2004 to 2007, he was employed by the Samsung SDI R\&D Center, Yongin, Korea, as a Senior Engineer. From 2007 to 2008, he was with the Department of Electrical Engineering, Hanyang University, as a Research Professor. Since 2008, he has been an Associate Professor with the School of Electronic and Electrical Engineering, Hongik University, Seoul, Korea. His current research interests include variable speed drives, power quality compensation devices, and power conversion systems for renewable energy sources. 